\title{
GENERALIZATION OF A THEOREM OF CLIFFORD
}

\author{
P. N. ÁNH
}

\begin{abstract}
The multiplicative monoid of principal ideals partially ordered by reverse inclusion, called the divisibility theory, of a Bezout $\operatorname{ring} R$ with one minimal prime ideal is a factor of the positive cone of a lattice-ordered abelian group by an appropriate filter if the localization of $R$ at its minimal prime ideal is not a field. This result extends a classical result of Clifford [6] saying that the divisibility theory of a valuation ring is a Rees factor of the positive cone of a totally ordered abelian group and suggests to modify Kaplansky's (later disproved) conjecture [8] as to a Bezout ring whose localization at every minimal prime ideal is not a field, is the factor of an appropriate Bezout domain.
\end{abstract}

\section{INTRODUCTION}

The divisibility theory of a Bezout ring $R$ with one minimal prime ideal is characterized in [4] as a Bezout monoid with one minimal m-prime filter. Inspired by Clifford's result [6] that the divisibility theory of a valuation ring is a Rees factor of the positive cone of a totally ordered abelian group, and by the existence of valuation rings which are not factors of valuation domains (see [8] for details), which is a negative answer to Kaplansky's conjecture, we extend Clifford's result to Bezout monoids with one minimal prime ideal. In addition, this extension suggests to sharpen Kaplansky's conjecture by asking for a classification of all factors of both Bezout domains and their divisibility theory, respectively.

A word about terminology. All structures are commutative. The divisibility theory of a ring is the monoid of principal ideals partially ordered by reverse inclusion. The set of nontrivial elements (i.e. those different from 1 and 0 ) of a subset $X$ in a monoid is denoted by $X^{\star}$. The 0 -extension of $X$ obtained by adding a new (extra) zero element to $X$ is denoted by $X^{\bullet}$.

\section{BASIC NOTIONS AND PRELIMINARY RESULTS}

For the benefit of the reader and the sake of self-containedness we present some basic definitions and results which can be found in full detail in [1], [2] and [4].

Definition 2.1 (cf. Definition 1.1 [1]). A Bezout monoid, in short B-monoid, is a commutative monoid $S$ with 0 which is a distributive lattice with respect to divisibility, called also the natural partial order $a \leq b \Longleftrightarrow a \mid b \Longleftrightarrow(\exists c: b=a c)$ such that multiplication is distributive over both meets and joins, and hyper-normality is satisfied, that is, for any $x, y$,

Date: Draft December 11, 2014.

2010 Mathematics Subject Classification. Primary: 06F05, 06F20; Secondary:13A05, 13 F05.

Key words and phrases. Bezout monoid, spectrum, prime filter, lattice-ordered abelian group.

The author was partially supported by both the Hungarian National Foundation for Scientific Research grants no. K-101515, Institute of Mathematics( Viện Toán Học 18 Hoàng Quôc Việt, Hà Nội) and VIASM (Vietnamese Institute of Advanced Study in Mathematics) for his stay in Hanoi, Vietnam. 
putting $d=x \wedge y \in S$ and $d x_{1}=x$ there is $y_{1}$ with $x_{1} \wedge y_{1}=1, d y_{1}=y$. A monoid with 0 is called 0 -cancellative if $a x=a y \neq 0 \Rightarrow x=y$.

For each $x \in S$ let $x^{\perp}=\{y \in S: x y=0\}$ denote the annihilator of $x$. If $x^{\perp}=0$, then $x$ is called a regular element or a non-zero-divisor. A filter is a subset $F$ of $S$ closed under $\wedge$ such that $a \in F, a \leq b \in S$ implies $b \in F$. A filter $F$ is called an m-prime filter if $a b \in F$ implies $a \in F$ or $b \in F$. A general theory of m-prime filters of B-monoids can be found in [1]. Filters and m-prime filters correspond naturally to ideals and prime ideals of rings, respectively.

By the main result in [4], the divisibility theories of Bezout rings with one minimal prime ideal is described as Bezout monoids with one minimal m-prime filter. The investigation of such B-monoids is initiated in [4]. We recall Propositions 3.1, 3.3, 3.5 and 3.6 as well as Corollary 3.4 of [4] as

Proposition 2.1. Let $S$ be a B-monoid with a smallest minimal m-prime filter $M, T=S \backslash M$, $Z=\{x \in S \mid \exists s \notin M: s x=0\} \subseteq M$ and $N=M \backslash Z$. Then $t<n<z$ for each $t \in T$, $n \in N, z \in Z ; T$ is the positive cone of a lattice-ordered abelian group $G$; and $Z M=0$.

Using the notation and assumptions of Proposition 2.1 one can take a classical localization $T^{-1} S$ of $S$ by inverting elements of $T$. Thus, $T^{-1} S$ is the set of pairs $(a, s)$ with $a \in T, s \in S$ subject to $(a, s) \sim\left(a_{1}, s_{1}\right)$ if there exists $a_{2} \in T$ with $a_{2} a_{1} s=a_{2} a s_{1}$. Notice that we have $(a, a s) \sim(b, b s)$ for all $a, b \in T, s \in S . T^{-1} S$ will be a distributive lattice-ordered monoid by putting

$(a, s)\left(a_{1}, s_{1}\right)=\left(a a_{1}, s s_{1}\right),(a, s) \wedge\left(a_{1}, s_{1}\right)=\left(a a_{1}, s a_{1} \wedge a s_{1}\right),(s, a) \vee\left(s_{1}, a_{1}\right)=\left(a a_{1}, s a_{1} \vee a s_{1}\right)$

We write $a^{-1} s$ for the equivalence class of $(a, s)$. It is a tedious but routine task to check that $T^{-1} S$ is well defined, i.e., independent of the choice of representatives, and then verify a flock of axioms. There is in addition a natural homomorphism from $S$ into $T^{-1} S$ sending $s \in S$ to $(a, a s)=a^{-1} a s\left(=s \in T^{-1} S\right), a \in T$. All elements of $S$ annihilated by some $a \in T$ are exactly the ones that go to $0 \in S_{M}$ under this homomorphism, i.e., $Z$ is the inverse image of $0 \in T^{-1} S$ in $S$. Moreover, if $r, s \in N$ map to the same (non-zero) element in $T^{-1} S$, then there is $a \in T$ such that $a r=a s$ holds. Therefore, by Proposition 2.23 [1] there is $z \in a^{\perp}$, i.e., $z \in Z$ satisfying $r \wedge z=s \wedge z$. By Proposition 2.1 one has $r=r \wedge z=s \wedge z=s$ whence $N$ maps injectively into $T^{-1} S$. Similarly, $T$ maps also injectively into $T^{-1} S$, i.e., $Y=S \backslash Z=T \cup N$ can be identified as a subset of $T^{-1} S$, although products of elements in $Y$ taken in $S$ can be in $Z$. It is worth noting that the induced partial order on $T^{-1} S$, similarly to the one on lattice-ordered groups, is not natural because of the existence of non-trivial invertible elements.

For any $a \in T$ and $s \in N$ there is $r \in N$ with $a r=s$ in view of Proposition 2.1, whence $a^{-1} s=a^{-1} a r=r \in N$. Hence $N^{\bullet}$ is in fact a filter of $T^{-1} S=X^{\bullet}$ where $X$ is the disjoint union $X=G \dot{\cup} N$. For any two $r, s \in N, d=r \wedge s$, one can write by the naturality of the partial order on $S$ together with hyper-normality $r=d r_{1}, s=d s_{1}$ with appropriate $r_{1}, s_{1} \in S$ satisfying $r_{1} \wedge s_{1}=1$. Consequently, at least one of $r_{1}, s_{1}$ is contained in $T$, i.e., the filters generated by $r, s$ in $T^{-1} S$ are comparable. This implies that the divisibility monoid of $T^{-1} S$, i.e., the monoid of principal filters of $T^{-1} S$ partially ordered by reverse inclusion is naturally order-isomorphic to the localization $\Sigma$ of $S$ at $M$ defined (see [1], Theorem 2.11) by putting elements of $T$ equal to 1 . We shall write $a^{\sigma}$ for the image in $\Sigma$ of $a \in S$, which can be considered also as the principal filter $T^{-1} S a$ of $T^{-1} S$ generated 
by $a$, and denote elements of $\Sigma$ by Greek letters $\alpha, \beta, \ldots$. Moreover, for each $\alpha \in \Sigma$ put $S_{\alpha}=\left\{b \in S \mid b^{\sigma}=\alpha\right\}$. Thus $S_{1}=T, S_{0}=Z$. Furthermore, for an element $s \in S$ we use also the notation $S_{s}=\left\{x \in S \mid x^{\sigma}=s^{\sigma}\right\}$. Therefore, for each $s \in N$ the subset $S_{s}$ can be considered as a copy of $G$ and $G$ acts naturally on $N$. The order in $\Sigma$ is preserved on $S$ in the sense that, for all $x, y \in S, x<y$ if $x^{\sigma}<y^{\sigma}$. In particular, if $x, y \notin Z$ satisfy $x y=y \neq 0$, then $x=1$. However, there are B-monoids with one minimal m-prime filter having elements $x \neq 1$ and $0 \neq y \in Z$ such that $x y=y$. The above arguments verify

Proposition 2.2. Assuming the conditions and notation of Proposition 2.1, $x y=y \notin Z$ implies $x=1$, the subset $Y=S \backslash Z$ of $S$ maps injectively into the classical localization $T^{-1} S$ of $S$ by inverting $T$. The filter of $T^{-1} S$ generated by $N$ is exactly $N^{\bullet}$. The quotient group $G$ of $T$ acts on $N$, the orbit of any $a \in N$ under this action is just $S_{a}$, a copy of $G$, consisting of elements of $T^{-1} S$ which are generators for the principal filter $T^{-1} S a$. In particular, the divisibility monoid of $T^{-1} S$ is precisely the localization $\Sigma$ of $S$ by the minimal m-prime filter $M$, and the order in $\Sigma$ is preserved on $S: x^{\sigma}<y^{\sigma} \Longrightarrow x<y$. Moreover, $T^{-1} S$ is $X^{\bullet}$ where $X$ is the disjoint union $X=G \dot{\cup} N$, and in the case $Z \neq M, Z$ is a factor of $G$ by an appropriate filter.

For another approach of Proposition 2.2 we refer to Theorems 3.9 and 3.10 [4]. More remarks on classical localization of B-monoids can be found before Proposition 2.29 of [1].

\section{The Generalization of Clifford's TheOREM}

¿From now on until Theorem 3.10 $S$ is a Bezout monoid with a smallest m-prime filter $M$ and $M \neq Z$, i.e., $\Sigma$ has at least three elements, although the equality $Z=0$ can happen. We use the notation of the previous section.

For a construction of the positive cone $P$ of a lattice-ordered abelian group $A$ such that $S$ can be represented as its factor, following Clifford's ideas [6], let $F(X)$ be the free associative monoid on the set $X=\left(T^{-1} S\right) \backslash\{0\}=G \dot{\cup} N$. Elements of $F(X)$ are words or, in another terminology, nonempty finite sequences $\hat{x}=\left\{x_{1}, \cdots, x_{m}\right\}$ of elements $x_{i} \in X$ where $m$ is called the length of $\hat{x}$, together with the empty word of the length 0 , and multiplication is juxtaposition: if $\hat{x}=\left\{x_{1}, \cdots, x_{m}\right\}$ and $\hat{y}=\left\{y_{1}, \cdots, y_{n}\right\}$, then $\hat{x} \hat{y}=\left\{x_{1}, \cdots, x_{m}, y_{1}, \cdots, y_{n}\right\}$.

The identity is obviously the empty word. Moreover, the evaluating map $\mathfrak{p}: \hat{x} \longmapsto \prod_{i=1}^{i=m} x_{i}$ is evidently a homomorphism from $F(X)^{\star}$, the subsemigroup of proper (=non-empty) words, onto $T^{-1} S$. It is important to emphasise now that $\mathfrak{p}$ is not a map into $S$; in fact, it is impossible to define natural maps from $F(X)$ into $S$. Throughout, by a word we always mean a proper word, and thus the identity of $F(X)$ is the improper (=empty) word.

A word $\hat{y} \in F(X)^{\star}$ is called a refinement of a word $\hat{x} \in F(X)^{\star}$ if $\hat{y}=\hat{y}_{1} \hat{y}_{2} \cdots \hat{y}_{m}=\prod_{i=1}^{i=m} \hat{y}_{i}$ with $\mathfrak{p}\left(\hat{y}_{i}\right)=x_{i}$ for all $i$. The relation "is a refinement of" is obviously transitive and reflexive, but not symmetric. Proposition 2.1 implies

Proposition 3.1. Let $\hat{x}=\left\{x_{1}, \cdots, x_{m}\right\}, \hat{y}=\left\{y_{1}, \cdots, y_{n}\right\}, \hat{z}=\left\{z_{1}, \cdots, z_{l}\right\}$ be words from $F(X)^{\star}$. If $\hat{y}$ and $\hat{y} \hat{z}$ are both refinements of $\hat{x}$, then $\mathfrak{p}(\hat{z})=1$.

Proof. Since $\hat{y}$ is a refinement of $\hat{x}$, there exists $i \leq n$ with $x_{1}=y_{1} \cdots y_{i}$. On the other hand, $\hat{y} \hat{z}$ is also a refinement of $\hat{x}$, whence either there exists $j \leq n$ with $x_{1}=y_{1} \cdots y_{j}$ or else $k$ such 
that $x_{1}=y_{1} \cdots y_{n} z_{1} \cdots z_{k}=\mathfrak{p}(\hat{y}) z_{1} \cdots z_{k}=\mathfrak{p}(\hat{x}) z_{1} \cdots z_{k}$. The second case implies $\mathfrak{p}(\hat{x}) \leq x_{1}$ and hence by the definition we have $0 \neq \mathfrak{p}(\hat{x})=\mathfrak{p}(\hat{y})=\mathfrak{p}(\hat{y}) \mathfrak{p}(\hat{z})$, which implies $\mathfrak{p}(\hat{z})=1$ by Proposition 2.2. In the first case, by symmetry one can assume $i \leq j$. Proposition 2.1 implies $x_{i+1} \cdots x_{j}=1$. Therefore letting $\hat{u}=\left\{x_{2}, \cdots, x_{m}\right\}, \hat{v}=\left\{y_{i+1}, \cdots, y_{n}\right\}$ one obtains that both $\hat{v} \hat{z}$ and $\hat{v}$ are refinements of $\hat{u}$, and then the result follows by induction on the length $n$ of $\hat{y}$.

Proposition 3.2. For words $\hat{x}=\left\{x_{1}, \cdots, x_{m}\right\}, \hat{y}=\left\{y_{1}, \cdots, x_{n}\right\}$ from $F(X)^{\star}$ there is a word $\hat{z}=\left\{z_{1}, \cdots, z_{l}\right\}$ of $F(X)$ such that exactly one of the following cases holds:

(1) $\hat{z}$ is a common refinement of $\hat{x}$ and $\hat{y}$;

(2) $\hat{z}$ is a refinement of $\hat{x}$ and there is $\hat{w} \in F(X)$ such that $\hat{z} \hat{w}$ is a refinement of $\hat{y}$;

(3) $\hat{z}$ is a refinement of $\hat{y}$ and there is $\hat{v} \in F(X)$ such that $\hat{z} \hat{v}$ is a refinement of $\hat{x}$.

Remark 3.1. Following Clifford, $\hat{z}$ will be called a greatest common part (GCP) of $\hat{x}$ and $\hat{y}$. Notice that if $x, y \in N$ satisfy $x^{\sigma}=y^{\sigma}$, then both $\hat{x}=\{x\}$ and $\hat{y}=\{y\}$ can be considered as greatest common parts of $\hat{x}, \hat{y}$. Therefore, greatest common parts are in general not unique!

Proof. Since $x_{1}^{\sigma}, y_{1}^{\sigma} \in \Sigma$ are comparable, by Proposition 2.2 exactly one of the following cases holds

(1) $x_{1}^{\sigma}<y_{1}^{\sigma}$, consequently $x_{1}<y_{1}$ whence $y_{1}=x_{1} \bar{y}_{1}$ holds for some $\bar{y}_{1} \in Y$. In that case put $z_{1}=x_{1}$ and $\hat{x}_{1}=\left\{x_{2}, \cdots, x_{m}\right\}, \hat{y}_{1}=\left\{\bar{y}_{1}, y_{2}, \cdots, y_{n}\right\}$

(2) $y_{1}^{\sigma}<x_{1}^{\sigma}$, consequently $y_{1}<x_{1}$ whence $x_{1}=y_{1} \bar{x}_{1}$ holds for some $x_{1} \in Y$. In this case put $z_{1}=y_{1}$ and $\hat{x}_{1}=\left\{\bar{x}_{1}, x_{2}, \cdots, x_{m}\right\}, \hat{y}_{1}=\left\{y_{2}, \cdots, y_{n}\right\}$;

(3) $x_{1}^{\sigma}=y_{1}^{\sigma}$ but $x_{1} \neq y_{1}$, then there is $1 \neq g \in G$ with $g x_{1}=y_{1}$. In this case put $z_{1}=x_{1}$ and $\hat{x}_{1}=\left\{x_{2}, \cdots, x_{m}\right\}, \hat{y}_{1}=\left\{g, y_{2}, \cdots, y_{n}\right\}$

(4) $x_{1}=y_{1}$. In this case put $z_{1}=x_{1}=y_{1}$ and $\hat{x}_{1}=\left\{x_{2}, \cdots, x_{m}\right\}, \hat{y}_{1}=\left\{y_{2}, \cdots, y_{n}\right\}$.

In each case $\left\{z_{1}\right\} \hat{x}_{1}$ and $\left\{z_{1}\right\} \hat{y}_{1}$ are refinements of $\hat{x}$ and $\hat{y}$, respectively. We now do exactly the same thing for the elements $\hat{x}_{1}$ and $\hat{y}_{1}$. We find elements $z_{2} \in X, \hat{x}_{2}, \hat{y}_{2} \in F(X)$ such that $\left\{z_{2}\right\} \hat{x}_{2},\left\{z_{2}\right\} \hat{y}_{2}$ are refinements of $\hat{x}_{1}, \hat{y}_{1}$, respectively. Consequently, $\left\{z_{1}, z_{2}\right\} \hat{x}_{2}=\left\{z_{1}\right\}\left\{z_{2}\right\} \hat{x}_{2}$ and $\left\{z_{1}, z_{2}\right\} \hat{y}_{2}=\left\{z_{1}\right\}\left\{z_{2}\right\} \hat{y}_{2}$ are refinements of $\hat{x}, \hat{y}$, respectively. Continuing this process, we construct a sequence of pairs of elements $\hat{x}_{i}, \hat{y}_{i} \in F(X)$, together with a sequence of elements $z_{i} \in X$ such that for each $i$ the elements $\left\{z_{1}, \cdots, z_{i}\right\} \hat{x}_{i},\left\{z_{1}, \cdots, z_{i}\right\} \hat{y}_{i}$ are refinements of $\hat{x}, \hat{y}$, respectively. The above process must terminate in a finite number $l$ of steps, for at each step at least one of the elements $\hat{x}_{i}, \hat{y}_{i}$ is shortened. Then $\hat{x}_{l}$ or $\hat{y}_{l}$, or both, is the empty word. Let $\hat{z}=\left\{z_{1}, \cdots, z_{l}\right\}$. Then $\hat{z} \hat{x}_{l}, \hat{z} \hat{y}_{l}$ are refinements of $\hat{x}, \hat{y}$, respectively. If both $\hat{x}_{l}$ and $\hat{y}_{l}$ are the empty word, we are in Case (1). If just $\hat{x}_{l}$ is the empty word, we are in Case (2). If just $\hat{y}_{l}$ is the empty word, we are in case (3).

Two words $\hat{x}, \hat{y} \in F(X)^{\star}$ are said to be similar, in symbol $\hat{x} \sim \hat{y}$, if they admit a common refinement. This relation is obviously reflexive and symmetric. We claim

Proposition 3.3. The relation $\sim$ is transitive.

Proof. Let $\hat{x}=\left\{x_{1}, \cdots, x_{m}\right\}, \hat{y}=\left\{y_{1}, \cdots, x_{n}\right\}, \hat{z}=\left\{z_{1}, \cdots, z_{l}\right\}$ be elements of $F(X)^{\star}$ such that $\hat{x} \sim \hat{y}$ and $\hat{y} \sim \hat{z}$. Let $\hat{u}$ be a common refinement of $\hat{x}$ and $\hat{y}$, and $\hat{v}$ be one of $\hat{y}$ and $\hat{z}$. By Proposition 3.2 and Remark 3.1 let $\hat{w}$ be a greatest common part of $\hat{u}$ and $\hat{v}$. If Case (1) holds, that is, if $\hat{w}$ is a common refinement of $\hat{u}$ and $\hat{v}$, then it is clearly also a common refinement of $\hat{x}$ and $\hat{z}$, too. Hence $\hat{x} \sim \hat{z}$. If Case (2) holds, that is, if $\hat{w}$ is a refinement of $\hat{u}$ 
and $\hat{w} \hat{a}$ is a common refinement of $\hat{v}$ for some $\hat{a} \in F(X)$, then $\hat{w}$ and $\hat{w} \hat{a}$ are both common refinements of $\hat{y}$. Therefore by Proposition $3.1 \mathfrak{p}(\hat{a})=1$. Consequently, $\hat{w} \hat{a}$ is also a common refinement of $\hat{u}$, henceforth it is a common refinement of $\hat{x}$ and $\hat{z}$ whence $\hat{x} \sim \hat{z}$. Case (3), of course, can be treated in a similar way.

Thus similarity is an equivalence relation in $F(X)^{\star}$. Consequently, one can see immediately that it is indeed a congruence relation. Let $H$ be the factor monoid of $F(X)^{\star}$ by this congruence. It will be convenient, however, to consider the words $\hat{x}, \hat{y}, \cdots$ of $F(X)^{\star}$ as themselves elements of $H$, with similarity as the equality relation.

Proposition 3.4. $H$ is commutative.

Proof. It suffices to see $\{x\}\{y\}=\{y\}\{x\}$ for all $x, y \in X$. There are three cases. If $x^{\sigma}<y^{\sigma}$, then $x<y$ whence $y=x z$ for some $z \in X$. Consequently, $\{x\}\{y\} \sim\{x\}\{z\}\{x\} \sim\{y\}\{x\}$. The case $y^{\sigma}<x^{\sigma}$ can be done in the same way. If $x^{\sigma}=y^{\sigma}$, then there is $g \in G$ with $y=g x$. Consequently, $\{x\}\{y\} \sim\{x\}\{g\}\{x\} \sim\{y\}\{x\}$, which completes the proof.

Proposition 3.5. $H$ is cancellative.

Proof. Let $\hat{x}=\left\{x_{1}, \cdots, x_{m}\right\}, \hat{y}=\left\{y_{1}, \cdots, x_{n}\right\}, \hat{z}=\left\{z_{1}, \cdots, z_{l}\right\}$ be elements of $F(X)$ such that $\hat{x} \hat{y} \sim \hat{x} \hat{z}$. Let $\hat{u}=\left\{u_{1}, \cdots, u_{k}\right\}$ be a common refinement of $\hat{x} \hat{y}$ and $\hat{x} \hat{z}$. Then there are $1 \leq i \leq j$ with $u_{1} \cdots u_{i}=x_{1}=u_{1} \cdots u_{j}$. This implies $u_{i+1} \cdots u_{j}=1$ in view of Proposition 2.2. Consequently, $\hat{u}_{1}=\left\{u_{i+1}, \cdots, u_{k}\right\}$ is a common refinement of $\hat{a} \hat{y}$ and $\hat{a} \hat{z}$ where $\hat{a}=\left\{x_{2}, \cdots, x_{m}\right\}$. The statement is now an immediate consequence of an obvious induction on the length $m$ of $\hat{x}$.

Corollary 3.6. 1 is the identity of $H$.

Proof. As usual we denote by 1 the word $\{1\}, 1 \in X$. By Proposition 3.5 the claim follows immediately from the similarity $\{x\}\{1\} \sim\{x\}$ for every $x \in X$.

Remark 3.2. It is worth noting that although $F(X)^{\star}$ does not have an identity, its factor $H$ does, and its identity element is not presented by the empty word.

Proposition 3.7. (1) If $\hat{x} \sim \hat{y}$ then $\mathfrak{p}(\hat{x})=\mathfrak{p}(\hat{y})$.

(2) If $\mathfrak{p}(\hat{x})=\mathfrak{p}(\hat{y}) \neq 0$ then $\hat{x} \sim \hat{y}$.

(3) If $\hat{x} \sim \hat{y} \hat{z}$ then $\mathfrak{p}(\hat{x})=\mathfrak{p}(\hat{y}) \mathfrak{p}(\hat{z})$.

Proof. (1) If $\hat{z}$ is a common refinement of $\hat{x}$ and $\hat{y}$, then $\mathfrak{p}(\hat{x})=\mathfrak{p}(\hat{z})=\mathfrak{p}(\hat{y})$ hold obviously.

(2) $\mathfrak{p}(\hat{x})=\mathfrak{p}(\hat{y})=z \in X$ imply that $\hat{x}$ and $\hat{y}$ are refinements of $\{z\}$, whence $\hat{x} \sim \hat{y}$.

(3) $\mathfrak{p}(\hat{y}) \mathfrak{p}(\hat{z})=\mathfrak{p}(\hat{y} \hat{z})=\mathfrak{p}(\hat{x})$.

Corollary 3.8. The map $x \in X \longmapsto\{x\} \in H$ is injective.

Proof. This assertion is a trivial consequence of Proposition 3.7 (1) and (2).

Let $F(Y)^{\star}$ be the subset of $F(X)^{\star}$ consisting of the words $\hat{x}=\left\{x_{1}, \cdots, x_{m}\right\}$ with $x_{i} \in$ $Y=S \backslash Z$. In particular, $F(Y)^{\star}$ is exactly the subsemigroup of proper words of the free associative monoid on the set $Y$. If $\hat{x} \in F(X)^{\star}$ contains some $x_{i} \in X$ satisfying $x_{i} \in N$, then the commutativity of $H$ as well as the fact that $g x_{i} \in N$ holds for all $g \in G$, imply that $\hat{x} \sim \hat{y}$ with $\hat{y} \in F(Y)$. This simple but very important observation allows us to reformulate Proposition 3.2 in the following stronger form. 
Proposition 3.9. For $\hat{x}=\left\{x_{1}, \cdots, x_{m}\right\}, \hat{y}=\left\{y_{1}, \cdots, x_{n}\right\} \in F(X)^{\star}$ there is $\hat{z}=\left\{z_{1}, \cdots, z_{l}\right\} \in$ $F(X)^{\star}$ such that exactly one of the following four cases holds:

(1) $\hat{z}$ is a common refinement of $\hat{x}$ and $\hat{y}$;

(2) $\hat{z}$ is a refinement of $\hat{x}$ and there is $1 \neq g \in G$ such that $\hat{z}\{g\} \sim \hat{y}$;

(3) $\hat{z}$ is a refinement of $\hat{x}$ and there is $\hat{w} \in F(Y)$ such that $\hat{z} \hat{w} \sim \hat{y}$;

(4) $\hat{z}$ is a refinement of $\hat{y}$ and there is $\hat{v} \in F(Y)$ such that $\hat{z} \hat{v} \sim \hat{x}$.

Remark 3.3. The fact that divisibility of $S$ does not induce a total order on $S$, causes huge of difficulties. Although we follow closely the proof of Clifford [6], we have to take a bigger set $X$ of free generators on which divisibility induces a total order although this new ordering does not coincide with the original partial order on $X$. This explains why we have to make a subtle and exhaustive path from Proposition 3.2 to this result. In particular, we are only interested in the monoid generated by $Y$ but to obtain its necessary properties we have to embed it in the bigger monoid $H$ generated by $X$. From now on we call the element $\hat{z}$ with the property described in Proposition 3.9 a greatest common part (GCP) of $\hat{x}$ and $\hat{y}$.

In view of Proposition 3.9 it is now easy to define a lattice structure on $H$. For words $\hat{x}=\left\{x_{1}, \cdots, x_{m}\right\}, \hat{y}=\left\{y_{1}, \cdots, x_{n}\right\} \in F(X)^{\star}$ let

(1) $\hat{x} \wedge \hat{y}=\hat{z}=\hat{x}=\hat{y}$ and $\hat{x} \vee \hat{y}=\hat{z}=\hat{x}=\hat{y}$ if $\hat{z}$ is a common refinement of $\hat{x}$ and $\hat{y}$;

(2) $\hat{x} \wedge \hat{y}=\hat{z}(1 \wedge g)$ and $\hat{x} \vee \hat{y}=\hat{z}(1 \vee g)$ if $\hat{z}$ is a refinement of $\hat{x}$ and there is $1 \neq g \in G$ such that $\hat{z}\{g\} \sim \hat{y}$

(3) $\hat{x} \wedge \hat{y}=\hat{z}=\hat{x}$ and $\hat{x} \vee \hat{y}=\hat{z} \hat{w}=\hat{y}$ if $\hat{z}$ is a refinement of $\hat{x}$ and there is $\hat{w} \in F(Y)^{\star}$ such that $\hat{z} \hat{w} \sim \hat{y}$;

(4) $\hat{x} \wedge \hat{y}=\hat{z}=\hat{y}$ and $\hat{x} \vee \hat{y}=\hat{z} \hat{v}=\hat{x}$ if $\hat{z}$ is a refinement of $\hat{y}$ and there is $\hat{v} \in F(Y)^{\star}$ such that $\hat{z} \hat{v} \sim \hat{x}$.

Since $G$ is a lattice-ordered abelian group with positive cone $T$, the above description of the greatest common part shows that $H$ is a disjoint union of copies of $G$ indexed by the positive cone of a totally ordered abelian group. This observation makes the verification of the following properties of $H$ a routine exercise. First, $H$ is in fact a distributive monoid and its positive cone, that is, the set of $\hat{x}$ satisfying $1=1 \wedge \hat{x}$, is just $P$, the image of $F(Y)^{\star}$ in $H$ under the congruence $\sim$. Moreover, the partial order induced by the lattice structure of $H$ on $P$ is clearly the natural one. Therefore, the quotient group $A$ of $P$ is an abelian lattice-ordered group whose positive cone is just $P$ (see also [3]).

We shall need the notion of Rees factor from semigroup theory, which is most conveniently expressed for Bezout monoids as follows.

Definition 3.4. Let $S$ be a B-monoid and $F$ be a filter in $S$ (hence $F$ is a semigroup ideal of $S$ ). Then the relation $x \sim y \Longleftrightarrow$ either $x=y \notin F$ or $x, y \in F$ is a congruence of the B-monoid $S$ called the Rees congruence of $S$ corresponding to $F$. The factor of $S$ by this congruence is denoted by $S / F$ and is called the Rees factor of $S$ by $F$.

We are now able to generalize Clifford's theorem.

Theorem 3.10 (cf. [6] Theorem 3). Let $S$ be a B-monoid with one minimal m-prime filter $M$ satisfying $M \neq Z=\{s \in S \mid \exists t \notin M: t s=0\}$. Then there exist an abelian lattice-ordered group $A$ and filters $B_{\infty} \subseteq C_{\infty}$ in the positive cone $P$ of $A$ such that

(1) $P$ is generated by the complement $P \backslash C_{\infty}$ of $C_{\infty}$ in $P$; 
(2) the Rees factors $P / C_{\infty}$ and $S / Z$ are isomorphic; consequently, $S$ is isomorphic to the Rees factor $P / C_{\infty}$ in the case $Z=0$;

(3) In the case $Z \neq 0, S$ is isomorphic to the factor of $P$ by $B_{\infty}$ in the lattice-theoretic sense, defined as $a \cong b \Longleftrightarrow \exists c \in B_{\infty}: a \wedge c=b \wedge c$.

Moreover, properties (1) and (2) above determine $P$ and $A$ uniquely to within an isomorphism leaving fixed the elements of $S \backslash Z$ if we identify $S \backslash Z$ with $P \backslash C_{\infty}$.

Proof. We take for $P$ and $A$ the monoid and its quotient group constructed before Definition 3.4 .

We have already seen that $A$ is an abelian lattice-ordered group having $P$ as its positive cone. The set $C_{\infty}$ of all $\hat{x}$ such that $\mathfrak{p}(\hat{x})=0$ is clearly a filter of $P$. Note that $\mathfrak{p}(\hat{x})=0$ implies trivially $\hat{x} \sim \hat{y}$ with $\hat{y} \in F(Y)$, i.e., $\hat{x} \in P$. Moreover, by Proposition 3.7, for any two elements $\hat{x}, \hat{y} \in P$ the equality $\mathfrak{p}(\hat{x})=\mathfrak{p}(\hat{y}) \neq 0$ holds if and only if $\hat{x} \sim \hat{y}$, that is, they represent the same element of $P$. Therefore $\mathfrak{p}$ induces an isomorphism between the Rees factors $P / C_{\infty}$ and $S / Z$, or equivalently, the mapping $\hat{x} \longmapsto \mathfrak{p}(\hat{x}) ; C_{\infty} \longmapsto 0$ is an isomorphism from $P / C_{\infty}$ onto $S / Z$. $P$.

It is obvious from the definition and the just verified identification that $P \backslash C_{\infty}$ generates

Now we are going to construct the filter $B_{\infty}$. We consider $P$ as the submonoid of $H$ generated by $Y$. Thus the elements of $P$ are given by sequences $\hat{y}=\left\{y_{1}, \cdots, y_{n}\right\}$ with $y_{i} \in Y=S \backslash Z$ subject to the congruence $\sim$. It is important to remark here that if $a, b \in$ $N ; g \in G$ satisfy $a b=0 \neq(g a) b=a(g b)$ in $S$, then although $\{g\}\{a, b\} \sim\{g a, b\} \sim\{a, g b\}$ represent the same element of $H$ and the latter two are (proper) words in $F(Y)^{\star},\{g\}$ is a proper word only in $F(X)^{\star}$. However, we claim that the evaluation map $\mathfrak{q}: P \longrightarrow S$ induced by multiplication sending $\hat{y} \in F(Y)^{\star}$ to $\mathfrak{q}(\hat{y})=\prod_{i=1}^{i=n} y_{i}$ is well defined, that is, it does not depend on the way of writing $\hat{y}$ as a product of elements from $Y$. Again it is very important here to emphasize that $\mathfrak{p}$ takes values in $T^{-1} S$ and $\mathfrak{q}$ takes values in $S$ and although $Y$ is a subset in both $T^{-1} S$ and $S$, multiplication of its elements depends strongly on where the results are considered. In fact, they give the same element in $Y$ if the corresponding value of $\mathfrak{q}$ is not contained in $Z$, otherwise the corresponding values of $\mathfrak{p}$ are always 0 meanwhile the ones of $\mathfrak{q}$ are (possibly nonzero) elements of $Z$. By this observation one can assume that $\mathfrak{q}(\hat{y})=\prod_{i=1}^{i=n} y_{i} \in Z$ and $\hat{y} \sim \hat{z}=\left\{z_{1}, \cdots, z_{l}\right\}$ with $z_{i} \in Y$. By Proposition 3.7 one has $\mathfrak{q}(\hat{z}) \in Z$. This implies that at least two of the $y_{i}$ as well as of the $z_{j}$ must belong to $N$. Moreover, by multiplying those $y_{i} \in T$ and $z_{j} \in T$, respectively, with one of the $y_{i} \in N$ and $z_{j} \in N$, one can assume that all $y_{i}$ and $z_{j}$ belong to $N$. Let $u_{1}=y_{1} \wedge z_{1} \in N, y_{1}=u_{1} t_{1}, z_{1}=u_{1} s_{1}, t_{1} \wedge s_{1}=1$. Then at least one of $t_{1}, s_{1}$ belongs to $T$. Therefore exactly one of the following three cases holds:

(1) if $t_{1} \in T, s_{1} \notin T$ let $\hat{y}_{1}=\left\{t_{1} y_{2}, \cdots, y_{n}\right\}$ and $\hat{z}_{1}=\left\{s_{1}, \cdots, z_{l}\right\}$;

(2) if $t_{1} \notin T, s_{1} \in T$ let $\hat{y}_{1}=\left\{t_{1}, y_{2}, \cdot, y_{n}\right\}$ and $\hat{z}_{1}=\left\{s_{1} z_{2}, \cdots, z_{l}\right\}$;

(3) if $t_{1}, s_{i} \in T$ let $\hat{y}_{1}=\left\{t_{1} y_{2}, \cdots, y_{n}\right\}$ and $\hat{z}_{1}=\left\{s_{1} z_{2}, \cdots, z_{l}\right\}$.

In each case $\mathfrak{q}\left(\left\{u_{1}\right\} \hat{y}_{1}\right)=\mathfrak{q}(\hat{y})$ and $\mathfrak{q}\left(\left\{u_{1}\right\} \hat{z}_{1}\right)=\mathfrak{q}(\hat{z})$. Since $A$ is a group, we obtain $\hat{y}_{1} \sim \hat{z}_{1}$. Hence induction on the sum $n+l$ shows that $\mathfrak{q}$ is indeed well defined. Consequently, $\mathfrak{q}$ is a surjective homomorphism between the B-monoids $P$ and $S$, and $B_{\infty}=\{a \in P \mid \mathfrak{q}(a)=$ 
$0\} \subseteq C_{\infty}$ holds trivially. If $a, b \in P$ satisfy $\mathfrak{q}(a)=\mathfrak{q}(b) \neq 0 \in S$ then we have also $\mathfrak{q}(a \wedge b)=$ $\mathfrak{q}(a \vee b)=\mathfrak{q}(a) \neq 0 \in S$. Therefore, one can assume without loss of generality that $a \leq b=a c$ for some $c \in P$. This implies $\mathfrak{q}(a)=\mathfrak{q}(b)=\mathfrak{q}(a) \mathfrak{q}(c)<0 \in S$, whence hyper-normality yields the existence of $s=\mathfrak{q}(d), s \in S$ with some $d \in P$ such that $1=s \wedge \mathfrak{q}(c)=\mathfrak{q}(c \wedge d)$ and $0=s \mathfrak{q}(a)=\mathfrak{q}(a d)$. The latter equality implies $a d \in B_{\infty}$. Taking into account that $\mathfrak{q}(\hat{x})=$ $1, \hat{x} \in F(Y)^{\star}$ if and only if $\hat{x}=\{1, \cdots, 1\}$, hence we obtain $c \wedge d=1 \in P$. Consequently, one has $a d \wedge a=a=a(c \wedge d)=a c \wedge a d=b \wedge a d$, which verifies claim (3).

To prove the uniqueness, let $A^{\prime}$ be another abelian lattice-ordered group together with its positive cone $P^{\prime}$ and filters $B_{\infty}^{\prime}, C_{\infty}^{\prime}$ satisfying the assumptions of Theorem 3.10. Identifying $P^{\prime} \backslash C_{\infty}^{\prime}$ with $S \backslash Z$ provides a surjective homomorphism from $F(Y)$ to $P^{\prime}$. This homomorphism factors through $P$ as is easy to check by the definition. To complete the proof, it suffices to show that the above homomorphism is injective. If two elements $a, b$ of $P$ map to the same element of $P^{\prime}$, then so do their join and meet. Thus one can assume $a \leq b=b c$ for some $c \in P$. But this implies that $c$ maps to $1 \in P^{\prime}$, whence $\mathfrak{p}(c)=1$. Consequently, $c=1$ in $P$ because 1 is the unique invertible element of the Rees factor $S / Z$. Hence $a=b$, showing the uniqueness of $A$ and $P$.

Remarks 3.5. (1) In case of a local B-semigroup $S$, i.e., in the case of a naturally totally ordered, 0-cancellative semigroup, Clifford [6] started with the free semigroup $F\left(S^{\star}\right)^{\star}$ not allowing any invertible elements, by taking advantage of the total order. Strange enough, in the general case we cannot start with $S^{\star} \backslash Z$ although at the end we have to use it to obtain a positive cone! Since we do not have a total order, even in the case of a local B-semigroup we start in fact by extending the set of free generators through inverting certain elements.

(2) Combining Therorem 3.10 with Clifford's result it follows immediately that the group $A$ in Theorem 3.10 is the direct product of a totally ordered abelian group with a lattice-ordered group $G$, endowed with the lexicographic order. However, this observation does not simplify the proof of Theorem 3.10 because it is unclear how the partial factor system of $S$ could be extended to one of $A$. On the other hand, in view of the fact that the lexicographic product of two nontrivial partially ordered groups is lattice-ordered iff the first is a chain and the second is a lattice, a possibly best generalization of Clifford's theorem is obtained in Theorem 3.10.

(3) Assume now that $S$ is a B-monoid with one minimal m-prime filter $M$ such that $M \neq Z=\{s \in S \mid \exists t \notin M: t s=0\} \neq 0$. In view of Proposition 2.1, for each $x \in N=M \backslash Z$ there is $y \in N$ such that $0 \neq x y=z \in Z$, whence together with Proposition 2.2, as in [4] Proposition 3.13, one can see easily that there is an involution, i.e., an order-reversing function $\imath: \Sigma^{\star} \longleftarrow \Sigma^{\star}, \imath^{2}=1$ such that $S_{\alpha} S_{\imath(\alpha)}=Z$. Moreover, it can be shown without difficulty that in the factor monoid of $H$ with respect to the congruence for which each occurrence of $G$ is a congruence class, all the words $\{d, g\}, d \in S_{\alpha}, g \in S_{\imath(\alpha)}, \alpha \in \Sigma^{\star}$ represent the same element, which is the greatest element not contained in $\Sigma^{\star}$. This fact together with Theorem 3.10 yields another proof for Claim 3 of [4] Theorem 3.15. One can also use Theorem 3.10 to represent B-monoids with one minimal m-prime filter of type II and III (see in [4]) as the divisibility theory of appropriate Bezout rings. In this case, these Bezout rings are factor rings of suitable Bezout domains. This fact shows again the advantage of using divisibility in solving certain ring problems. 
(4) If $S$ is a B-monoid such that $S \backslash\{0\}$ is the positive cone of a lattice-ordered group $G$, then $S$ is obviously the factor of the positive cone of the lexicographic product $\mathbb{Z} \oplus G$ by the filter generated by the subset $\{(n, g) \mid n \geq 0\}$, where $\mathbb{Z}$ is the additive group of integers.

Let $S$ be a B-monoid with one minimal m-prime filter $M$ and $Z=\{s \in S \mid \exists t \notin M: t s=$ $0\}$. Then $Z$ is a Bezout act (see the definition in [4] Definition 3.1) over $T^{\bullet}, T=S \backslash M$. By Proposition $2.2 Z$ is the factor of the quotient group $G$ of $T$ by the congruence $g_{1}, g_{2} \in G$ : $g_{1} \sim g_{2} \Longleftrightarrow \exists h \in F: g_{1} \wedge h=g_{2} \wedge h$ with respect to an appropriate filter $F$ of $G$. The group $\mathbb{Z} \oplus G$, where $\mathbb{Z}$ is the additive group of integers, is lattice-ordered with respect to the order $(m, g)<(n, h)$ iff either $m<n$ or $m=n, g<h$. Then $P=\{(n, g) \mid n \geq 0\}$ is the positive cone of $\mathbb{Z} \oplus G$ and $S$ is obviously the factor of $P$ with respect to the filter generated by the subset $\{(1, g) \mid g \in F\}$ in case $M=Z$, and $Z$ is the factor of $G$ by the filter $F$. Therefore Theorem 3.10 can be reformulated in a sharpened form as follows.

Theorem 3.11. Let $S$ be a B-monoid with one minimal m-prime filter $M$ such that $Z=$ $\{s \in S \mid \exists t \notin M: t s=0\}$ is a factor of the quotient group of $T=S \backslash M$. Then $S$ is a factor of the positive cone of a lattice-ordered abelian group.

If a B-monoid $S$ with one minimal m-prime filter $M$ satisfies $M=\{s \in S \mid \exists t \notin M$ : $t s=0\}=Z \neq 0$, then it is an open question whether $S$ is a factor of the positive cone of a lattice-ordered abelian group. In view of counterexamples to Kaplansky's conjecture on valuation rings (cf. [8] and references given there) the answer can be negative and of a set-theoretic nature. Hence it is important and interesting to find sufficient conditions for a positive answer, i.e., conditions on B-monoids $S$ with one minimal m-prime filter $M$ satisfying $M=\{s \in S \mid \exists t \notin M: t s=0\}=Z \neq 0$ to be factors of positive cones of appropriate latticeordered abelian groups. If one assumes in addition that the filter $F$ generated by all $a^{\perp}$ $\left(a \in Z^{\star}\right.$ ) is proper, i.e., $F \neq S$, then $S$ is a submonoid of a classical localization $U^{-1} S$ where $U$ is the complement in $S$ of some m-prime filter of $S$ containing $F$. Since $U \subseteq T$ and $T=S \backslash M$ is cancellative, m-primeness implies that the subset $\left\{u^{-1} v \mid u, v \in U\right\}$ is a latticeordered abelian subgroup $L$ of $G$, the quotient group of $T$. Since the divisibility theory $\Omega$ of $U^{-1} S$ is totally ordered, interchanging the role of $G, H$ and $\Sigma, \Omega$, respectively, a repetition of the proof of Theorem 3.10 yields, in view of [1] Proposition 2.29, the following result.

Theorem 3.12. Let $S$ be a B-monoid with one minimal m-prime filter $M$ with $M=\{s \in$ $S \mid \exists t \notin M: t s=0\} \neq 0$. If the filter generated by all $a^{\perp}\left(a \in M^{\star}\right)$ is proper, then $S$ is a factor of the positive cone of a lattice-ordered abelian group. More generally, if $I$ is an $m$-prime filter in an arbitrary B-monoid $S$, and $K=\{s \in S \mid \exists t \notin I: t s=0\}$, then the factor B-monoid $S / K$ is a factor of the positive cone of a lattice-ordered abelian group.

\section{REFERENCES}

1. P. N. Ánh, L. Márki, P. Vámos, Divisibility theory in commutative rings: Bezout monoids, Trans. Amer. Math. Soc. 364 (2012), 3967 - 3992.

2. P. N. Ánh, M. Siddoway, Divisibility theory of semi-hereditary rings, Proc. Amer. Math. Soc. 138 (2010), $4231-4242$.

3. P. N. Ánh, M. Siddoway, Gauss' Lemma and valuation theory, submitted.

4. P. N. Ánh, M. Siddoway, Bezout monoids with one minimal m-prime filter, submitted.

5. G. Birkhoff, Lattice theory, AMS Colloquium Publ. 25, 3rd ed., 3rd printing 1979. 
6. A. H. Clifford, Naturally totally ordered commutative semigroups, Amer. J. Math. 76 (1954), $631-646$.

7. L. Fuchs, Partially ordered algebraic systems, Pergamon Press 1963.

8. L. Fuchs and L. Salce, Modules over non-Noetherian domains, Mathematical Surveys and Monographs 84, AMS, 2001.

9. T. S. Shores, On generalized valuation rings, Michigan Math. J. 21 (1974), $405-409$.

Alfréd Rényi Institute of Mathematics, Hungarian Academy of Sciences, 1364 Budapest, PF. 127, Hungary

E-mail address: anh.pham.ngoc@renyi.mta.hu 